\title{
The Self-excitation Process in Electrical Rotating Machines Operating in Pulsed Power Regime
}

\author{
M.D. Driga \\ The University of Texas Department of Electrical and Computer Engineering \\ S.B. Pratap, A.W. Walls, and J.R. Kitzmiller \\ The Center for Electromechanics at The University of Texas at Austin
}

\begin{abstract}
The self-excitation process in pulsed air-core rotating generators is fundamental in assuring record values of power density and compactness. This paper will analyze the conditions for the self-excitation process in pulsed electrical machines used as power supplies for electromagnetic launchers, using the analogy and methods of the positive feedback in control systems.

In the classical "ferromagnetic" electrical machines in the self-excitation process, the machine output is used in order to gradually excite the generator until the steady-state voltage is finally reached. The end of the process and, consequently, the stability and repeatability, is assured by the intersection of the strongly nonlinear magnetization characteristic and the excitation straight line.

In the air-core, pulsed power machines such as homopolar generators and compulsators, the two characteristics are apparently linear and both the end and the initiation of the process must be forced. The paper shows that the pattern of the self-excitation process is very similar in all the EML power supplies (homopolar, compulsators, asynchronous) and the initially unstable process reaches stability in a very controlled way.
\end{abstract}

The paper also shows how the self-excitation can create a new EML power supply with an original use, from a classical electrical machine.

\section{INTRODUCTION}

$\mathrm{T}^{\mathrm{s}}$ HE invention of the self-excitation process in electrical machines credited to Werner von Siemens almost a century and a half ago, looked (and still does) somewhat a miracle. For an electric generator considered as a power amplifier with a power gain equal to the ratio of the output to the excitation power, the self-excitation starts with an extremely small input (the remnant magnetism, for example) and reaches the full output power, increasing manifold the one-stage power gain. The amplification is obviously achieved on the account of the energy supplied by the prime mover. In [1] as in a majority of classical treatments of electrical machines is stated the general idea that "any selfexcitation is inconceivable without the magnetic saturation, since no stability of voltage and current in the machine would be attained. Without saturation... the magnetic flux and simultaneously the voltage and current would rise so excessively that the machine would burn out...”

This statement is incomplete, since the machine does not burn out if the excessive currents are flowing during a relatively short pulse - intensity and duration being in competition - but involuntarily suggests the use of selfexcitation in pulsed rotating, electric generators for EML technologies in which the pulse duration is measured in milliseconds. Of course, even in EML pulsed rotating power supplies, the runaway self-excitation may be stopped by fault conditions such as mechanical failure from excess braking force, increased ohmic losses from heating and even depletion (or insufficient) rotational kinetic energy stored (quantified by the critical $\omega=\omega_{c}$ ).

This paper tries to show that the self-excitation is one of the fundamental ways of increasing the power densities for such generators, thus drastically reducing their weight and volume. As an illustration, Fig. 1 presents a picture of the Cannon Caliber Electromagnetic Gun (CCEMG) generator a self-excited compulsator of high power density and compactness capable of delivering 1.2 $\mathrm{MJ}$ to a railgun for three five-round salvos at a short repetition rate of $5 \mathrm{~Hz}$. The self-excited compulsator stores $40 \mathrm{MJ}$ at $12,000 \mathrm{rpm}$ and weighs only 2,045 kg, its compactness being shown by a graphic comparison [2, 3].

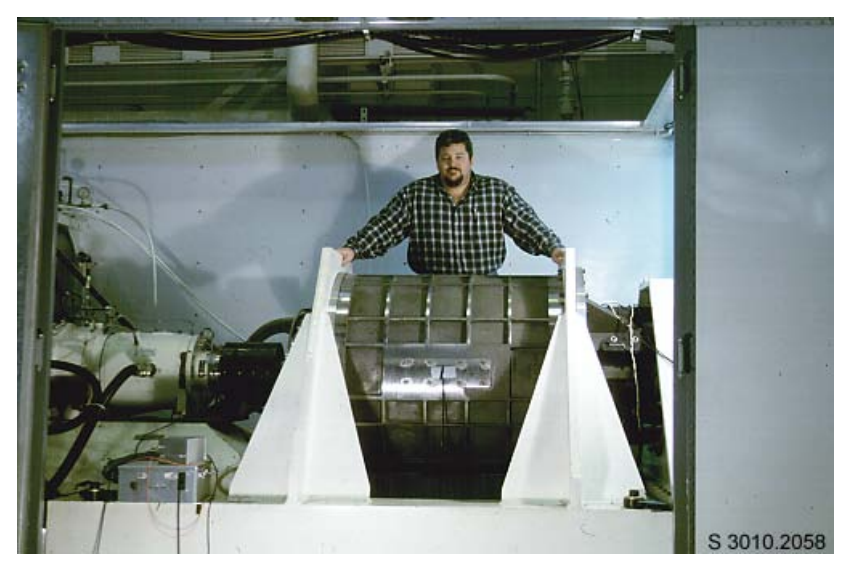

Fig. 1. Cannon caliber self-excited compulsator 
This paper also points out that the self-excitation is a closed loop process which, unlike in classical control theory, employs a positive feedback: the energy of the electric generator is brought back to the excitation stage using the positive feedback network in order to achieve the desired terminal voltage and other output parameters in conditions of a relatively high amplification factor.

Also, while self-excitation can be applied to all types and topologies of electrical machines (homopolar and heteropolar, synchronous and asynchronous, steady-state and pulsed operation), it has become evident lately that it offers incredible benefits, especially to the pulsed rotating generation used in electromagnetic launch technology. Such benefits are two-fold: not only does it achieve, almost exponentially (due to the positive feedback connection), high values of the excitation flux densities, but such values are maintained, only for the short duration of the machine discharge time, limiting the excitation losses to reasonable values.

The basic topologies and types of electrical machines will be considered and discussed, showing that in principle (or rather in spirit), all their self-excitation processes are similar: their point of departure is a small magnetic field (a seed) such as remnant magnetism, a discharging capacitor, etc., leading to an unstable (positive feedback!) initial exponential increase of the output. However, nearing the final state, a limiting mechanism occurs, either due to a drop in the angular velocity below the critical value $\left(\omega_{c}\right)$ as in the case of homopolar generators, as a result of a massive conversion of kinetic energy into magnetic energy, or by a skillful commutation as in the compulsators case, or by a stable exponential approach to the steady-state enforced by saturation in iron core dc generators.

Finally, a new generator or, conversely, a new beginning for an electrical machine with almost no applications outside of the area of pulsed EML is proposed as an ideal power supply for industrial/civilian applications.

\section{THE ROLE OF SELF-EXCITATION IN INCREASING POWER DENSITY}

In order to quantify the role of self-excitation in the increase of power density, we will refer to Figure 2, showing the reversibility principle in electromechanical energy conversion for a unit volume of active conductor (or by multiplication by the volume element $d v$, for an elementary volume of active conductor). This diagram makes the point that an electromechanical structure represents a pulsed generator or an accelerator, depending on the direction of the power flow. In Fig. 2, $P_{k i n}, P_{c o n v}, P_{e l}$ are the densities for kinetic, converted, and electrical power (in $w / \mathrm{m}^{3}$ ), while $P_{\text {mech }}$ and $J^{2} / \sigma$ represent the mechanical and electrical (joule) losses (also expressed in $w / \mathrm{m}^{3}$ ). In spite of the appearance of being incomplete and simplistic, the diagram describes the local power density for the converter. Such local power density in the condition of lossless electromechanical power conversion per unit volume of active conductor of the generator is given by the mixed vector product:

$$
-(J \times B) \bullet \bar{u}=(\bar{u} \times \bar{B}) \bullet \bar{J}
$$

where $J$ is the current density $\left(A / \mathrm{m}^{2}\right), B$ is the magnetic flux density $(\mathrm{T})$, and $\bar{u}$ is the local velocity.

When the two sides of expression (1) are multiplied by the elementary volume $d v$, we obtain the true definition of instantaneous conversion. The "active conductor" is the seat of the power conversion and by the principle of equality of action and reaction, is equally felt in both the stator and rotor of the electromechanical converter.

The flux density $\bar{B}$ in expression (2) is the vectorial sum of the impressed excitation field $\bar{B}_{i m p}$ and the induced field by the armature reaction, $\bar{B}_{a, \text { ind }}$ (invoking the Lenz rule) such that:

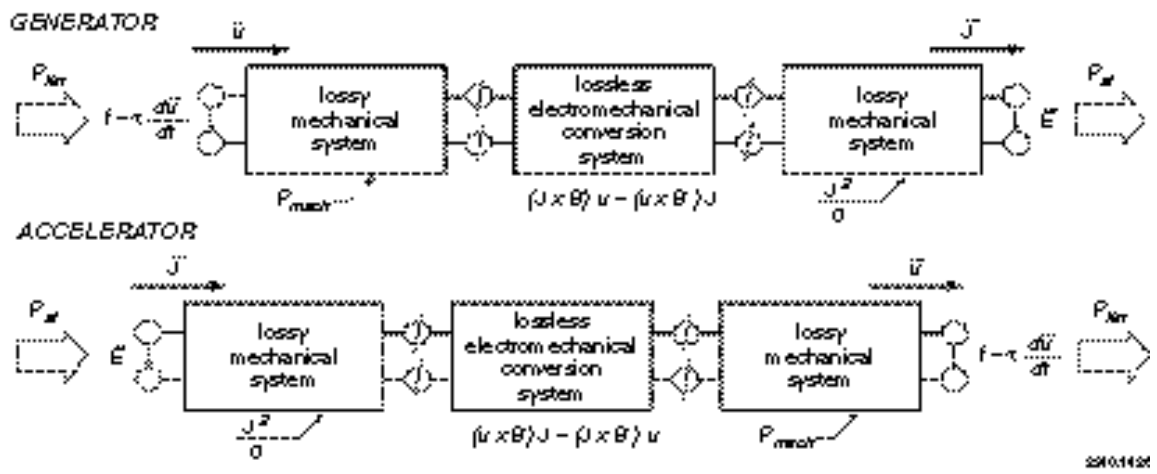

Fig. 2. The principle of reversibility in EML electromechanical conversion 


$$
\left[-\bar{J} \times\left(\bar{B}_{i m p}-\bar{B}_{a, \text { ind }}\right)\right] \bullet \bar{u}=\left[\bar{u} \times\left(\bar{B}_{i m p}-\bar{B}_{a, \text { ind }}\right)\right] \bullet \bar{J}
$$

A pulsed electrical machine exploits the manner in which the two $\bar{B}$ vectors add (or rather subtract) in different moments of discharge. By partially eliminating the direct axis shield, one can achieve a first self-excitation scheme without unreasonably increasing the losses by eddy currents, especially if a separate exciter winding is used in order to "protect" the optimality of the main armature winding.

The mixed vector product in expressions (1) and (2) points out that increasing the excitation field $B$ by self-excitation is one of the three fundamental directions of drastically reducing the weight and volume of the rotation power supplies used in electromagnetic launch technology: (a) the increase in the velocity $\bar{u}$, by employing the most advanced composite structural materials for the rotor; (b) the increase in the current density $\bar{J}$ by improvements in the machine cooling; and (c) the increase in $B$ by self-excitation up to the limit allowed by the losses in the short excitation time.

Formula (2) cautions us that the impressed excitation field $B_{\text {imp }}$ will give an optimistic evaluation of the power density; the reaction field (mainly armature reaction) may diminish it and the skills of the designer are used to achieve the best compromise.

\section{SELF-EXCITATION AS A CLOSED LOOP, POSITIVE FEEDBACK MECHANISM}

As was defined above, self-excitation is a term describing a closed loop process in which the energy of an electric generator is brought back to the excitation stage using a positive feedback mechanism in order to achieve the desired terminal voltage and, consequently, the desired output parameters in conditions of a relatively high amplification factor. Fig. 3 shows a basic, unilateral feedback loop. Obviously, the schemes for self-excitation are more complex, but this basic scheme makes the main point of the positive feedback, where the summing junction (comparator) adds, rather than subtracts, the relatively small input with the output signal $C$ modified by element $B$ [5].

In this paper, the closed loop characterization is introduced not as an interesting analogy, but mainly leading to a methodology of design and analysis of the selfexcitation using the Root Locus Method. The

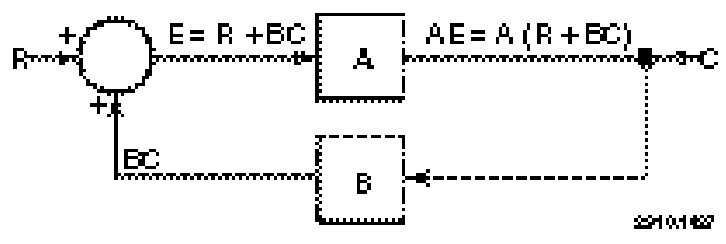

Fig. 3. Closed loop with positive feedback. characteristic equation of the self-excited rotating electrical generator modeled in Fig. 3 is:

$$
1+A B(s)=0 \text { (3)Equation (3) leads to several relations }
$$

for the analysis and design for the generator, since it implies two conditions related to magnitude and phase angle:

$$
|A B(s)|=1 ; \quad<A B(s)=(2 k+1) \pi
$$

The rules for root locus construction, well known from the control system theory, cannot be used here because of positive feedback, which leads to instability.

For the self-excited electrical machines, the rules will be modified such that: (a) the gain $K$ is varied from 0 to $+\infty$ (instead of taking negative values as in the case of regular, negative feedback); (b) root loci occur on a segment of real axis, only if there are an even number (as opposed to an odd number for negative feedback) or poles and zeros of the open loop transfer function lying to the right of that segment; (c) finally, $(2 k+1) \pi$ is replaced in construction by $2 k \pi$.

\section{SELF-EXCITATION MECHANISMS IN PULSED ROTATING GENERATORS USED IN EML TECHNOLOGY}

A decade ago, the words EML technology were synonymous with EML for military applications and still are for a majority of ultra-high performance devices. However, the electromagnetic launch (EML) technology has matured and, lately, extended from the strictly military applications to many civilian industrial and manufacturing applications today. One of the most successful is, for example, a new thermal spray process where, using an augmented railgun, electromagnetic forces can accelerate powder particles to velocities more than double that of existing technologies which are limited by the thermodynamic expansion of gases. The new EML processes allow fusion bonding of greater strength, as well as improved coating density.

In the following is shown that the self-excitation mechanisms for different pulsed power rotating machines have very similar differential equations and the three stages mentioned at the beginning of the paper exist in all of them: initiation magnetic field, unstable exponential increase of voltage or current, stable approach to the final output value.

\section{A. Self-Excited Homopolar Generator}

The self-excited homopolar generator (HPG) used for EML and related technologies has an air-core magnetic system and uses the self-excitation in order to achieve a reasonable output voltage $[6,7,8]$.

The output voltage of the machine (volts) is:

$$
V=\alpha I \omega
$$

where $I$ is the current (A), $\omega$ is the angular velocity ( $\mathrm{rad} / \mathrm{sec}$ ), and $\alpha$ is a generator constant. If $R$ is the total resistance of the HPG system $(\Omega)$, then the condition of selfexcitation $\frac{d I}{d t} / I>0$ occurs only if

$$
\omega>\omega_{c}
$$


where

$$
\alpha \omega_{c}=R
$$

$\omega_{c}$ being the "critical" angular velocity.

The differential equation describing the electric circuit is:

$$
L \frac{d I}{d t}-(\alpha \omega-R) I=0
$$

Showing that beginning with a starting (seed) excitation magnetic field, the current will have an initial unstable exponential increase:

$$
I=I_{0} e^{+\frac{\alpha \omega-R}{L} T}
$$

In fact, the HPG velocity is decreasing as the machine is converting kinetic into magnetic energy. The conservation of energy is written as:

$$
\frac{1}{2} J\left(\omega^{2}-\omega_{0}^{2}\right)+\frac{1}{2} L I^{2}+\int_{0} I^{2} R d t=0
$$

$\omega_{0}$ being the initial velocity of the generator, and $J$ the moment of inertia of its rotor.

Then the maximum current (Fig. 4) is approximately:

$$
I_{m} \approx\left(\omega_{0}-\omega_{c}\right) \sqrt{\frac{J}{L}}
$$

the time to peak current:

$$
t_{0}=\frac{L / 12}{\left(\omega_{0} / \omega_{c}\right)-1} \ln \left(2 \frac{I_{m}}{I_{0}}\right)
$$

while the efficiency of the system [Knoepfel(8)], assuming a load inductance $L_{L}(H)$ is:

$$
\eta_{H}=\frac{L_{L} I_{m}^{2}}{J \omega^{2}}
$$

\section{B. Self-Excited Air-Core Compulsators}

Self-excited air-core compulsators represent the most promising power supplies for EML technologies. The example given in this paper (fig. 1) is a self-excited air-core 4-pole, rotating armature compact compensated pulsed alternator - size compatible with the Amphibious Assault Vehicle. Its objective was to power an EML system capable of launching three five-round salvos of 185 grams launch packages to $1.85 \mathrm{~km} / \mathrm{sec}$ at a firing rate of $5 \mathrm{~Hz}$. Self-excited, very compact compulsators are developed for a multitude of field-based EM launchers.

The compulsators represent optimized electromechanical converters capable of many refinements, leading to high compactness and efficiencies. However, in a simplified approach, they still retain the three basic stages of the selfexcitation. The first one is achieved by capacitor initiation module. The second one, representing the unstable exponential increase of the excitation, is described by a differential equation similar to the one in the previous paragraph.

$$
L_{f} \frac{d i_{f}}{d t}-\left(\alpha \omega-R_{f}\right) i_{f}=0
$$

with the solution

$$
i_{f}=i_{f 0} e^{+\frac{\alpha \omega-R_{f}}{L_{f}}}
$$

As was mentioned previously, the third stage leading to the stable approach to the full excitation is achieved by a solidstate commutation. Also, since the parameters of the armature winding are not optimal for self-excitation, a separate exciter winding is used in order to achieve the optimal matching, leading to a minimum charging time.

As shown by formula (2) at the beginning of this paper, the compensation winding produces a magnetic field which subtracts from the excitation field. A thorough treatment of the limitation of the charging time for the field coil is given in [4]. Figs. 5 and 6 show the field current transients and normalized charging losses, respectively, for several values for the shield thickness.

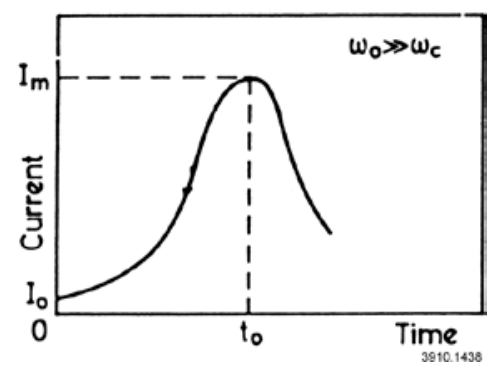

Fig. 4. Self-excitation in an air-core homopolar generator

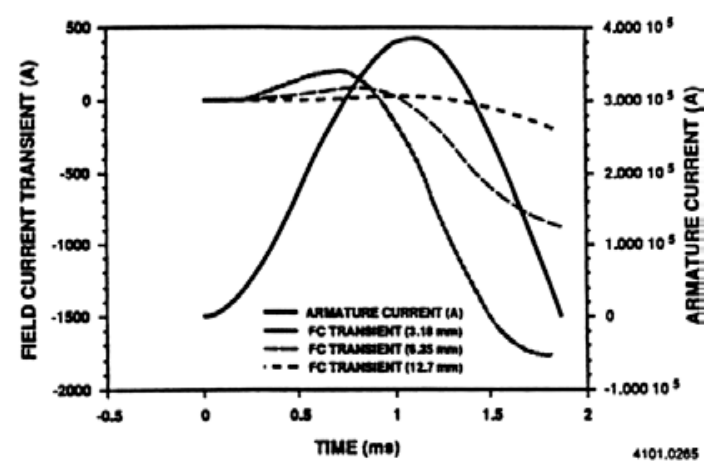

Fig. 5. Excitation current transient for different shield thickness

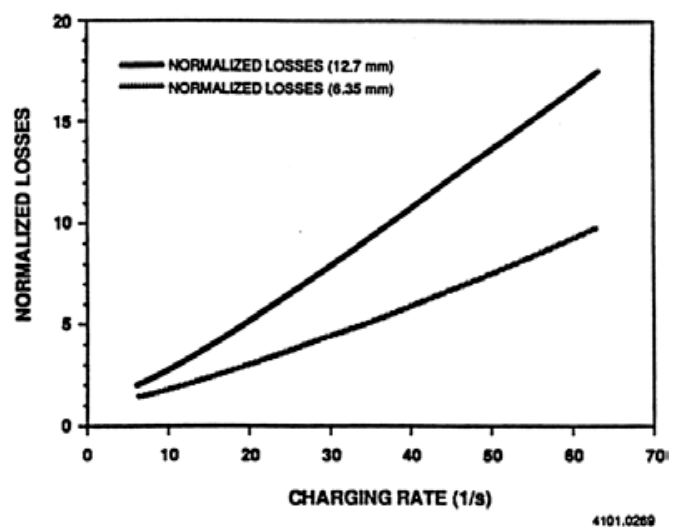

Fig. 6. excitation losses for different shield thickness 


\section{Asynchronous generators for pulsed power}

Asynchronous generators for pulsed power require a much more complex system of self-excitation, since they involve an ac current with a rigid phase shift and magnitude in relation to the generator current. The capacitors providing the reactive power of excitation are prohibitively large. However, the equations governing the self-excitation process are strikingly similar to the ones for other types of electric generators.

Condition for self-excitation:

$$
R=\omega G \beta>0
$$

Differential equation for the process:

$$
\frac{d^{2} i_{m}}{d t^{2}}-\frac{\left(\omega G \beta-R_{m}\right)}{L} \frac{d i_{m}}{d t}+\frac{i_{m}}{L C}=0
$$

where $R$ is the resistance of the system, $L$ and $C$ are the inductance and capacitance, respectively,

$$
L=G\left(L_{1}+\alpha\right)
$$

being the apparent inductance.

The back electromotive force is $E=\omega G \beta I_{m}$. The selfexcitation of asynchronous pulsed generator as an EML power supply is an extremely complex subject and its treatment will be the object of a future, follow-up paper.

\section{Self-excitation for dc Machines}

The self-excitation for dc machines is used for the steadystate and does not bring the manifold increase in power density, due to the inherent limitations of the iron-core magnetic circuit. It brings, however, stability to the operation (fig. 7).

The excitation process, the variable electromotive force in the armature of the dc machine induced by the magnetic flux $(\Phi)$ is identical to the terminal voltage $v$ which, in turn, is balanced at every moment by the ohmic drop on the resistance $R$ or the excitation circuit:

$$
v=N \frac{d \Phi}{d t}+R i
$$

$$
\begin{gathered}
\text { or } \\
\Delta v=v-R i=N \frac{d \Phi}{d t}
\end{gathered}
$$

It is the difference of voltage ( $\Delta v)$ between the magnetization curve and the ohmic resistance line which drives the self-excitation process until the steady-state operation is reached. Since the terminal voltage $(v)$ of the machine is always proportional to the flux:

$$
\frac{k \omega \Phi}{k \omega \Phi_{0}}=\frac{v}{V}
$$

and we can write (see fig. 8)

$$
\begin{aligned}
& \frac{d \Phi}{d t}=\frac{\Phi_{0}}{V} \frac{d v}{d t} \\
& \quad \text { and } \\
& \Delta v=\frac{N \Phi_{0}}{V_{x}} \frac{d v}{d t}=T \frac{d v}{d t}
\end{aligned}
$$

$T$, the excitation time constant (sec), is an absolute constant for a given machine.

The time of self-excitation is given by what is called numerical excitation time, described extremely simply:

$$
t=\frac{N \Phi_{0}}{V} \int \frac{d v}{\Delta v}
$$

The graphical calculations shown in Fig. 8 (a,b,c,d) depict the evolution of self-excitation as known from the classical theory of electrical machines and reproduced here since they are similar in spirit to the pattern of self-excitation evolution

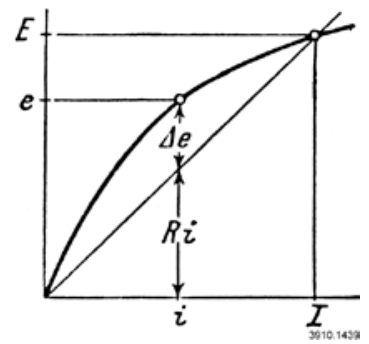

Fig. 7. D.C. self-excitation in DC "ferromagnetic" generator

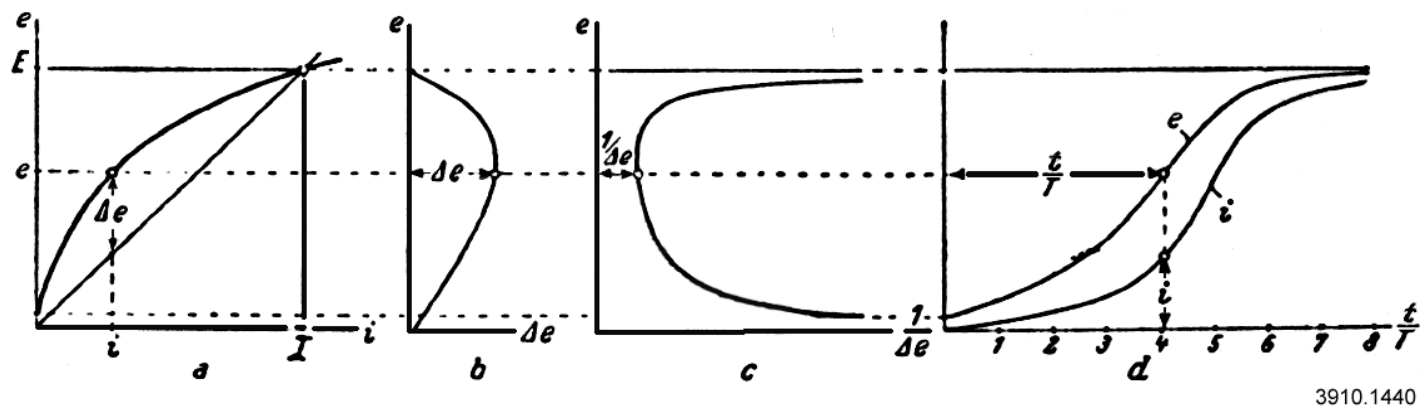

Fig. 8. Current self-excitation in DC machines, numerical integration, unstable and stable stages (a, b, c, d) 
for the less traditional power supplies and for EML technologies considered in the previous paragraph. In (1), the plots of the self-excitation evolution in relative values versus time show that they consist of two distinct segments: (a) first, an unstable exponential increase of the voltage of the type $v=V e^{+\beta t}$

(b) a second one as a stable approach to the steady-state of the form $v=V\left(1-e^{-\beta t}\right)$

The first one (a) occurs in the initial part of the selfexcitation of all the pulsed electrical machines (homopolar, compulsator, dc heteropolar). The second one (b) is imposed by the massive conversion of energy in homopolar generators, by solid-state commutation and interruption in compulsators, or occurs naturally, due to the strong nonlinearity of the characteristic of magnetization.

\section{A DC SELF-EXCITED SERIES GENERATOR AS AN IDEAL POWER SUPPLY FOR A HYPERVELOCITY PLASMA SPRAYER}

Probably a place where the self-excitation in context of EML technology may be used with spectacular results is the dc series generator, a machine which has no applications in everyday technology, but can serve as the ideal power supply for the augmented railgun used successfully as a hypervelocity plasma sprayer [9]. The plasma in the railgun discharge is accelerated early to a snowplow velocity of 4000 $\mathrm{m} / \mathrm{sec}$ and subsequent energy is used to gather more mass of Inconel powder into the gas column. The energetics of the process allow fusion bonding of greater strength than created by customary low velocity processes (limited to $1 \mathrm{~km} / \mathrm{sec}$ by thermodynamic considerations) and markedly improved coating density.

The dc series generator, due to its self-excitation particularities, represents an ideal power supply, matching extremely well the characteristics demanded by the hypervelocity augmented railgun plasma accelerator. Closing

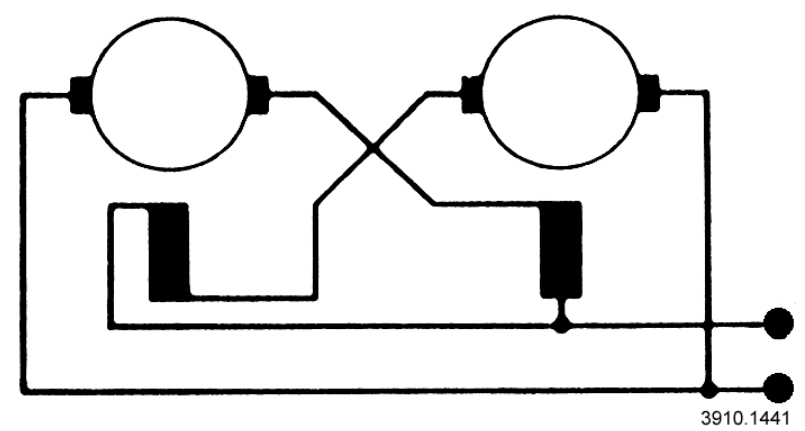

Fig. 9. Series excitation pair of power supplies for EML powder deposition the generator armature on the practical short-circuit of the railgun starts and leads to self-excitation. For such generator, the no-load characteristic must be calculated as for the separately excited machine, since at no-load, the excitation winding (in series) does not have current. The emf induced in the armature is found with the normal tools and procedures known from the classical electrical machine theory. In this kind of generator (series excitation - self-excited) to every point of the no-load characteristics corresponds a uniquely defined load current flowing through both excitation and armature windings. Fig. 9 shows a connection which will be used in powering the railgun plasma spray device. The selfexcitation of both series generators connected parallel is balanced by placing each excitation winding in the armature circuit of the other generator.

Motoring up the generator is done as, of course, a series motor having the advantage of outstanding characteristics of such a drive at an almost ( $T \omega=$ constant $)$ constant power.

\section{CONCLUSION}

The self-excitation process plays in EML rotating pulsed rotating generators an even stronger role in performance enhancement than in their classical counterparts. The paper shows that the process has the same stages in all the machine topologies, the difference being in the artificial ending of the unstable stage in the air-core machines, as opposed to the automatic transition to stability in the iron-core machines. A modification of the Root Locus Method for positive feedback systems is advanced as a design method of self-excited aircore systems.

\section{REFERENCES}

[1] R. Rudenberg. Transient Performance of Electric Power Systems, The MIT Press, 1970.

[2] M.D. Werst, C.E. Penney, T.J. Hotz, and J.S. Kitzmiller, "Continued testing of the cannon caliber electromagnetic gun system (CCEMB)," IEEE Transactions on Magnetics, vol. 35, No. 1, January 1999, pp. 388-393.

[3] M.D. Driga, S.B. Pratap, and W.F. Weldon, "Advanced Compulsator Design," IEEE Transactions on Magnetics, vol. 25, No. 1, January 1989, pp. 149-156.

[4] S.B. Pratap, "Limitation on the minimum charging time for the field coil of air core compensated pulsed alternators," IEEE transactions on Magnetics, vol 33, January 1997, pp. 495-501.

[5] B.C. Kuo, Automatic Control Systems, Prentice Hall, 1992.

[6] Heinz Knoepfel, Pulsed High Magnetic Fields, North Holland Publishing Co., 1970.

[7] T. Bodefeld and H. Sequenz, Electrical Machinery, Springer 1981 (in German).

[8] Richter, Rudolph. Electrical Machines, Birkhauser Verlag, 1976 (in German).

[9] R.C. Zowarka, et. al., Electromagnetic Powder Deposition Experiments. IEEE Transactions on Magnetics, vol. 35, No. 1, January 1999, pp. 512-517. 